\title{
Unusual Mammographic and Ultrasound Findings in a Patient With Ductal Carcinoma in Situ (DCIS)
}

\author{
Gudrun Peters ${ }^{\mathrm{a}, \mathrm{b}}$, Catherine M Jones ${ }^{\mathrm{a}}$
}

\begin{abstract}
Ductal carcinoma in situ (DCIS) is a non-invasive, normally asymptomatic, form of breast cancer which is increasingly detected on breast imaging. The typical mammographic finding for DCIS is microcalcification, and rarely is it seen on breast ultrasound. However in a minority of patients with isolated DCIS, palpable changes on clinical examination and/or mass-like findings on mammogram and ultrasound are seen in the absence of an invasive breast cancer. This case report illustrates an atypical example of symptomatic histologically proven DCIS with mass-like changes on breast imaging.
\end{abstract}

Keywords: Breast; Symptomatic DCIS; Mammography; Ultrasound

\section{Introduction}

Ductal carcinoma in situ (DCIS) is a non-invasive breast cancer with neoplastic cells contained inside the ductal system. Before the introduction of mammographic screening, DCIS accounted for less than $5 \%$ of breast cancers [1]. Currently DCIS accounts for $25-33 \%$ of all screen detected malignancies and $5 \%$ of symptomatic cancers [2]. As DCIS is not usually clinically palpable and does not present with visible breast changes, most cases are found on mammography as microcalcifications [3]. Other changes, such as mass lesions,

Manuscript accepted for publication March 13, 2012

${ }^{a}$ Regional Imaging Tasmania, 49 Augusta Road, Lenah Valley 7008 TAS, Australia

${ }^{\mathrm{b}}$ Corresponding author: Gudrun Peters, Regional Imaging Tasmania, 49

Augusta Road, Lenah Valley 7008 TAS, Australia.

Email: Gudrun.Peters@i-med.com.au

doi:10.4021/jmc639e ductal changes, and architectural distortion have also been described [4]. Rarely, DCIS can present as a palpable mass, nipple discharge, or Paget's disease [5]. The following case report presents unusual mammographic and ultrasound appearances of a symptomatic DCIS case.

\section{Case Report}

An 81 year old woman was initially referred by her general practitioner for radiological investigation of short term amnesia, weight loss and fullness of the left iliac fossa. The patient was otherwise healthy and did not have a history of malignancy.

Contrast computer tomography (CT) scans of the head and abdomen were performed. The cerebral CT was unremarkable but on the abdominal study, a mixed density, partially cystic, mass in the right breast was incompletely visualised. Interstitial changes and ground-glass opacity of the posterobasal segment of the right lower lobe were also described.

In light of these findings, the patient was referred by the general practitioner to a breast surgeon for clinical examination and a CT chest was performed for further evaluation of the basal lung changes. On clinical examination, the patient presented with two firm but mobile lumps in the right breast. One mass was felt in the outer central breast and a second lump was palpated in the upper inner quadrant.

\section{Imaging}

Corresponding to this, two heterogeneous complex right breast masses were identified on the CT chest (Fig. 1). The solid components showed contrast enhancement. Dependent atelectatic changes were seen in the lung bases, otherwise no significant changes were noted; specifically, no parenchymal nodules, lymphangitic carcinomatosa, pleural effusion, or nodal enlargement.

Standard two view mammography (craniocaudal (CC) and medio-lateral oblique (MLO)) showed several mass lesions on the right in fatty replaced breast tissue. The two largest mass lesions (Fig. 2) corresponded to the palpable 

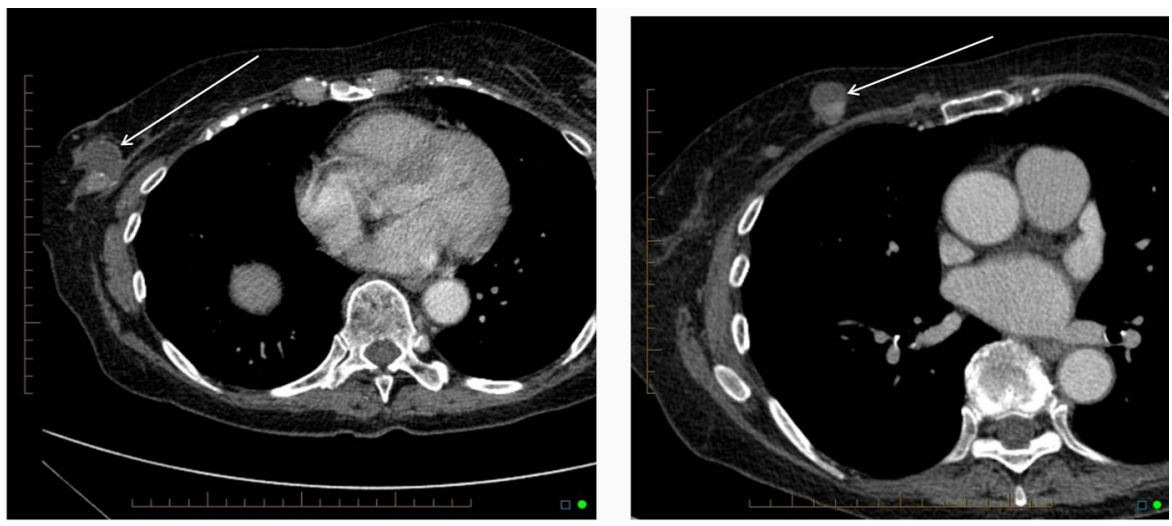

Figure 1. CT scan of the chest showing two partially cystic, partially solid mass lesions in the right breast.

lumps, were well circumscribed and contained coarse and pleomorphic calcifications. The largest lesion in the central slightly outer breast measured $48 \mathrm{~mm}$ in maximum diameter. The second largest mass in the upper breast, prepectoral region measured $31 \mathrm{~mm}$ in maximum diameter and was not visalized on the $\mathrm{CC}$ view due to its posterior position.

The other smaller mammographic abnormalities were not palpable.

Ultrasound of both breasts and the right axilla were performed. Corresponding to the two clinical and mammographic mass lesions, two lesions with solid and cystic components were noted on ultrasound. Color doppler showed blood vessels within the solid components. Lesion 1 was seen at the 9 o'clock position, $40 \mathrm{~mm}$ from the nipple and measured $41 \mathrm{~mm}$ in maximum diameter (Fig. 3a). Lesion 2 was localized at the 2 o'clock position, $70 \mathrm{~mm}$ from the nipple and measured $27 \mathrm{~mm}$ in maximum diameter (Fig. $3 c)$. In addition there were several small simple cysts scattered throughout the right breast, thought to correlate to the smaller mammographic abnormalities. No abnormality was seen in the axilla.

Aspiration of the cystic component of lesion 1 and ultrasound-guided $16 \mathrm{G}$ core biopsies of the solid components of lesion 1 and 2 were peformed. The aspirate featured malignant glandular epithelial cells consistent with primary ductal carcinoma (either invasive or in situ). The histopathology
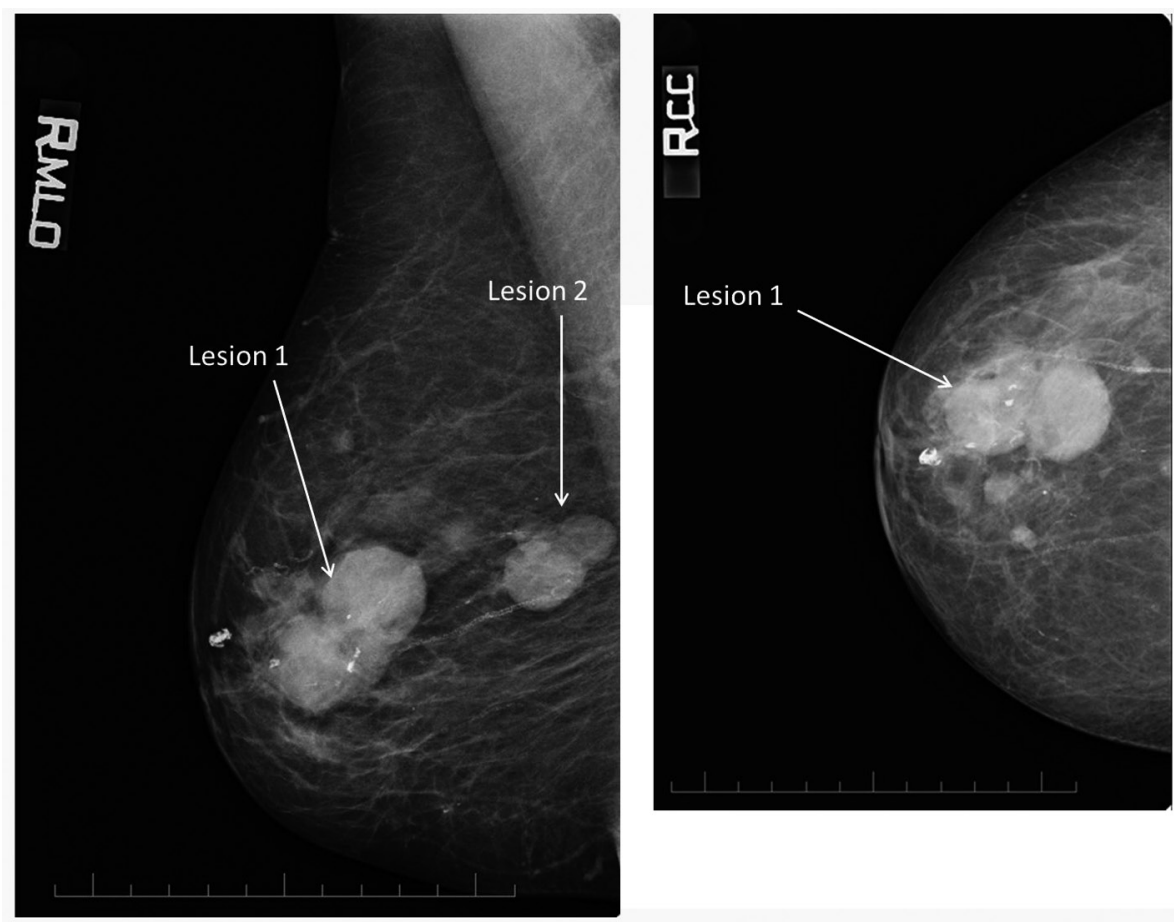

Figure 2. Right medio-lateral oblique and craniocaudal view. The two largest mass lesions (lesion 1 and lesion 2) correspond to the palpable lumps. 

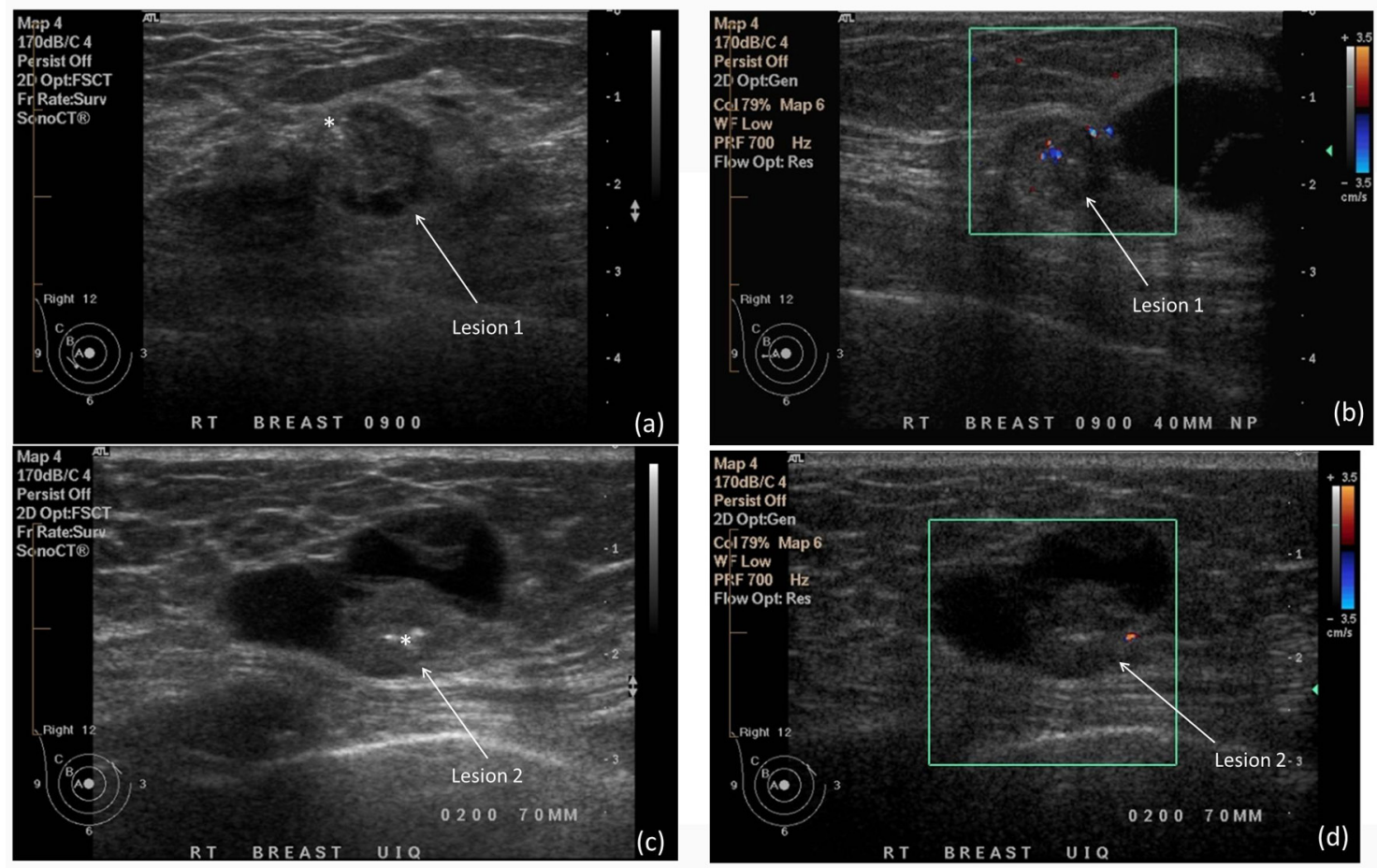

Figure 3. A partially solid, partially cystic mass with fairly well-circumscribed margins at the 9 o'clock position (Lesion 1, a) and 2 o'clock position (Lesion 2, c). Hyperechoic foci within the solid components of both lesions $\left(^{*}\right)$ indicate coarse calcifications. (b) and (d) Color Doppler imaging shows internal blood flow within the solid components of the lesions.

result of the core biopsies showed high grade DCIS without invasion.

The patient underwent a right mastectomy and sentinel node biopsy. Final histopathology confirmed high grade calcifying DCIS, with solid, cribriform and papillary patterns and cystic change. The sentinel lymph nodes were negative.

\section{Discussion}

The majority of DCIS cases are asymptomatic and are diagnosed from screening mammography. Rarely, DCIS is symptomatic and presents with a palpable lump. This patient presented with weight loss, and was found to have two palpable masses which were proven to be DCIS with no invasive ductal adenocarcinoma component. The weight loss, amnesia and abdominal pain are not explained by the DCIS finding and remain a diagnostic dilemma.

The mammographic appearances in this patient are atypical for DCIS. The primary finding is multiple mass lesions, with limited microcalcification. Foci of coarse calcification were also seen, which is atypical. Typical findings of pure DCIS on mammography are microcalcification with no mass lesion. Ultrasound is usually normal, and it used to exclude an underlying invasive component and axillary nodal involvement.

Fortunately for this patient, the mass lesions were identi- fied on an abdominal CT study, and further breast imaging was performed. The ultimate diagnosis of high grade DCIS with no invasive component or evidence of metastatic disease has been curatively treated with a right mastectomy, required due to the multicentricity and large size of the masses. She remains at higher risk of contralateral breast cancer and will undergo more rigorous screening than the usual population.

This case highlights the variety of appearances of DCIS on mammography and ultrasound, as well as a rare clinically apparent breast mass subsequently proven to be pure DCIS.

\section{Disclosures}

No financial or ethical disclosures.

\section{References}

1. D’Orsi CJ. Imaging for the diagnosis and management of ductal carcinoma in situ. Journal of the National Cancer Institute. Monographs. 2010;2010(41):214-217.

2. Berg WA, et al. Diagnostic Imaging. Breast. 1st ed. Canada: Amirsys, 2008.

3. Yang WT, Tse GM. Sonographic, mammographic, and histopathologic correlation of symptomatic ductal carci- 
noma in situ. AJR. American journal of roentgenology. 2004;182(1):101-110.

4. Hofvind S, Iversen BF, Eriksen L, Styr BM, Kjellevold K, Kurz KD. Mammographic morphology and distribution of calcifications in ductal carcinoma in situ diagnosed in organized screening. Acta radiologica.
2011;52(5):481-487.

5. Shin HJ, Kim HH, Kim SM, Kwon GY, Gong G, Cho OK. Screening-detected and symptomatic ductal carcinoma in situ: differences in the sonographic and pathologic features. AJR. American journal of roentgenology. 2008;190(2):516-525. 